\title{
THE
}

\section{Multicomponent folate-targeted magnetoliposomes: design, characterization, and cellular uptake}

\author{
Geoffrey D. Bothun \\ University of Rhode Island, gbothun@uri.edu \\ Alline Lelis \\ University of Rhode Island \\ Yanjing Chen \\ University of Rhode Island \\ Kyle Scully \\ University of Rhode Island

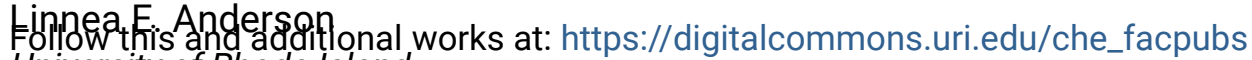 University of Rhode Island
Part of the Chemical Engineering Commons

This is a pre-publication author manuscript of the final, published article.

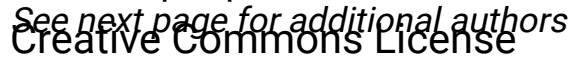

\section{(c) (1) (9)}

This work is licensed under a Creative Commons Attribution-Noncommercial-No Derivative Works 4.0 License.

\section{Citation/Publisher Attribution}

Bothun, G. D., Lelis, A., Chen, Y., Scully, K., Anderson, L. E., \& Stoner, M. A. (2011). Multicomponent folatetargeted magnetoliposomes: design, characterization, and cellular uptake. Nanomedicine:

Nanotechnology, Biology and Medicine, 7(6), 797-805. doi: 10.1016/j.nano.2011.02.007

Available at: https://doi.org/10.1016/j.nano.2011.02.007

This Article is brought to you for free and open access by the Chemical Engineering at DigitalCommons@URI. It has been accepted for inclusion in Chemical Engineering Faculty Publications by an authorized administrator of DigitalCommons@URI. For more information, please contact digitalcommons-group@uri.edu. 
Authors

Geoffrey D. Bothun, Alline Lelis, Yanjing Chen, Kyle Scully, Linnea E. Anderson, and Matthew A. Stoner 


\title{
Multicomponent folate-targeted magnetoliposomes: Design, characterization, and cellular uptake
}

\author{
Geoffrey D. Bothun ${ }^{\dagger,}{ }^{\star}$, Alline Lelis ${ }^{\dagger, \ddagger}$, Yanjing Chen ${ }^{\dagger}$, Kyle Scully $\ddagger$, and Matthew A. Stoner ${ }^{\ddagger}$ \\ tDepartment of Chemical Engineering, University of Rhode Island, 16 Greenhouse Road, \\ Kingston, RI 02881 \\ ‡Department of Biomedical and Pharmaceutical Sciences, University of Rhode Island, 41 Lower \\ College Road, Kingston, RI 02881
}

\section{Abstract}

Multifunctional folate-targeted cationic magnetoliposomes (FTMLs) have been prepared with coencapsulated doxorubicin (DOX) and anionic superparamagnetic iron oxide (SPIO) nanoparticles with $5 \mathrm{~nm} \gamma-\mathrm{Fe}_{2} \mathrm{O}_{3}$ cores and $16 \mathrm{~nm}$ hydrodynamic diameters. Nanoparticle encapsulation (89\%) was confirmed by cryogenic transmission electron microscopy, and the presence of the oppositely charged nanoparticles did not cause liposome aggregation. The FTMLs had an average diameter of $174 \pm 53 \mathrm{~nm}$ and existed as unilamellar and cup-shaped liposomes, which was attributed to dissimilar lipid packing parameters and the presence of PEG-lipids. A 3-fold increase in DOX release was achieved over two hours when the encapsulated SPIO nanoparticles were heated by an alternating current electromagnetic field operating at radiofrequencies (RF). Results with human cervical cancer cells (HeLa), which have been shown to exhibit high folate receptor (FR) expression, confirmed FTML surface binding and cellular uptake. In contrast, no uptake was observed for lower FR-expressing human breast carcinoma cells (ZR-75-1).

\section{Keywords}

Magnetoliposome; SPIO nanoparticle; Folate receptor; PEG-lipid

\section{Introduction}

\begin{abstract}
Magnetoliposomes, which are self-assembled liposomes containing encapsulated magnetic nanoparticles, are one promising example of a multifunctional liposome-nanoparticle assembly (LNA). ${ }^{1-4}$ Their multi-functionality stems from the ability to guide them in vivo using magnets, to utilize the particles as image contrast agents, to provide hyperthermia treatment by heating the nanoparticles in an alternating current electromagnetic field at radiofrequencies (i.e. RF-heating), and to provide liposomal drug delivery (see reviews by De Cuyper and Joniau ${ }^{5}$ and Soenen et $\mathrm{al}^{6}$ ). A lesser-explored approach related to this work is utilizing localized RF-heating within the magnetoliposomes for controlled drug release. ${ }^{7-10}$ Headgroup-functionalized lipids with targeting or stabilizing moieties (e.g. polyethylene glycol or PEG) can incorporated within the bilayer to target specific receptors
\end{abstract}

(C) 2011 Elsevier Inc. All rights reserved.

Corresponding author: bothun@egr.uri.edu, +1-401-874-9518.

Publisher's Disclaimer: This is a PDF file of an unedited manuscript that has been accepted for publication. As a service to our customers we are providing this early version of the manuscript. The manuscript will undergo copyediting, typesetting, and review of the resulting proof before it is published in its final citable form. Please note that during the production process errors may be discovered which could affect the content, and all legal disclaimers that apply to the journal pertain. 
overexpressed on cancer cell surfaces and to increase bioavailability, respectively. Cationic lipids can also be used to bind anionic molecules or aid cell uptake. ${ }^{11,12}$

Magnetoliposomes are promising, but do have shortcomings. There is evidence that inorganic nanoparticles, including iron oxide, can inhibit cell function. ${ }^{13,}{ }^{14}$ Thus, it is advantageous to reduce the concentration of encapsulated nanoparticles and maximize cellular targeting. This presents a challenge as high nanoparticle concentrations are needed to, for example, achieve sufficient MRI imaging contrast, hyperthermia, and magnetic guidance. For hyperthermia or RF-induced drug release, the energy absorbed by the particles is converted into heat through magnetic losses via Brown and Néel relaxation. To achieve liposome release via permeabilization at low nanoparticle concentrations or low electromagnetic field strengths, the heating should be localized near the bilayer surface. This can be achieved by chemically or physically binding the nanoparticles to the bilayer.

We report the design and characterization of folate-targeted cationic magnetoliposomes (FTMLs) containing encapsulated doxorubicin (DOX; Figure 1). Folate receptor (FR) is overexpressed in many cancer cells compared to healthy cells and exhibits a high affinity for folate conjugates $\left(K_{\mathrm{d}} \sim 100 \mathrm{pM}\right) .{ }^{15},{ }^{16}$ FTMLs were prepared based on the premise that electrostatic attraction between the cationic lipids and anionic superparamagnetic iron oxide (SPIO) nanoparticles would enhance physical bilayer-nanoparticle binding and, with RFheating, this binding would trigger transbilayer release. This compliments our recent work on focused nanoparticle heating achieved by embedding small hydrophobic SPIO nanoparticles within liposomal bilayers. ${ }^{9}$ SPIO nanoparticles with $16 \mathrm{~nm}$ hydrodynamic diameters were selected for their high curvature, which favored nanoparticle-liposome binding rather than the formation of supported lipid bilayers. The objective of this work was to characterize FTML structure and morphology, and to demonstrate RF-tiggered DOX release and cellular uptake by cervical cancer cells (HeLa).

\section{Experimental}

\section{Materials}

Cholesterol and the lipids 1,2-dioleoyl-sn-glycero-3-phosphocholine (DOPC), 1,2dipalmitoyl-3-trimethylammonium-propane (DPTAP), 1,2-dipalmitoyl-sn-glycero-3phosphoethanolamine-N-(7-nitro-2-1,3-benzoxadiazol-4-yl) (NBD-DPPE), 1,2-dimyristoylsn-glycero-3-phosphoethanolamine-N-[methoxy(polyethylene glycol)-750] ( $\mathrm{PEG}_{750^{-}}$ DMPE), and 1,2-distearoyl-sn-glycero-3-phosphoethanolamine- $\mathrm{N}$-[folate(polyethylene glycol)-2000] (Fol-PEG 2000 -DSPE) were purchased from Avanti Polar Lipids (Alabaster, AL). Doxorubicin (DOX) was purchased from Sigma-Aldrich (St. Louis, MO). Phosphate buffered saline (PBS, 1 or $10 \times, \mathrm{pH}$ 7.4) was prepared using sterile deionized ultrafiltered water obtained from a Millipore Direct 3-Q purification system (Billerica, MA).

Maghemite SPIO nanoparticles ( $5 \mathrm{~nm} \gamma-\mathrm{Fe}_{2} \mathrm{O}_{3}$ cores) were purchased from Ocean NanoTech (Springdale, AR). The particle surfaces contained oleic acid capped by an amphiphilic polycarboxylate coating that was formed by hydrolyzing poly(maleic anhydride-alt-1-octadecane) (Figure 2C). ${ }^{17}, 18$ The nanoparticles had a hydrodynamic diameter of $16 \pm 3 \mathrm{~nm}$, based on dynamic light scattering, and a zeta potential of $-39.8 \mathrm{mV}$ (Malvern Instruments Zetasizer Nano ZS, results not shown).

\section{Folate-targeted cationic magnetoliposome (FTML) formation}

FTMLs were prepared at $10 \mathrm{mM}$ total lipid by thin film hydration followed by extrusion through double-stacked polycarbonate membranes with $200 \mathrm{~nm}$ pore diameters. The bilayer components used to prepare the FTMLs are shown in Table 1. In this process, aliquots of the lipids and cholesterol dissolved in chloroform were pipetted into test tubes to provide the 
desired composition. Nitrogen gas was flowed over the samples to evaporate the chloroform and leave dry lipid films. Residual solvent was removed from these films by vacuum drying at $635 \mathrm{~mm} \mathrm{Hg}$ for $2 \mathrm{~h}$. The dry lipid films were hydrated at room temperature with either $10 \times$ PBS buffer (FTLs) or 10 $\times$ PBS containing both SPIO nanoparticles and DOX (FTMLs). SPIO nanoparticles were encapsulated at a concentration of $1 \mu \mathrm{M}\left(6 \times 10^{14}\right.$ particles $\mathrm{ml}^{-1}$ or $0.19 \mathrm{mg} \gamma-\mathrm{Fe}_{2} \mathrm{O}_{3} \mathrm{ml}^{-1}$ ) and DOX was encapsulated at $2 \mu \mathrm{M}$. Samples were dialyzed for $24 \mathrm{~h}$ using $1000 \mathrm{kDa}$ tubular cellulose acetate membranes with stirring and frequent media exchange to remove unencapsulated DOX. DOX removal after dialysis was determined by fluorescence spectroscopy (Perkin Elmer LS55) against a calibration curve of DOX in PBS. A third sample was prepared within the FR targeting lipid (MLs; no Fol-PEG 2000 -DSPE) for cellular uptake studies. All samples were blanketed with nitrogen, stored in the dark at room temperature, and used within 2 weeks of preparation.

Nanoparticle encapsulation efficiency was determined immediately after sample preparation using a centrifugation procedure similar to that of Pradhan et al. ${ }^{19}$ However, in our case the cations in PBS screened the repulsive charges between unencapsulated nanoparticles and led to aggregation. The sample was centrifuged at $1000 \mathrm{~g}$ for $10 \mathrm{~min}$ at $4{ }^{\circ} \mathrm{C}$. The precipitate containing nanoparticle aggregates was resuspended in DI water, digested in nitric acid, and analyzed by inductively coupled plasma mass spectrometry (ICP-MS, Thermo Electron X Series, Waltham, MA). The reported encapsulation efficiency is based on the 57Fe isotope.

\section{Cryogenic transmission electron microscopy (cryo-TEM)}

The size and structure of the FTLs and FTMLs were analyzed by cryo-TEM (JEOL JEM-2100F, Peabody, MA). Samples were prepared at room temperature and $100 \%$ relative humidity using a Vitrobot (FEI Company), which is a PC-controlled robotic system for sample vitrification. Quantifoil grids were used with $2 \mu \mathrm{m}$ carbon holes on 200 square mesh copper grids (Electron Microscopy Sciences, Hatfield, PA). To prepare a cryo-TEM sample for analysis, a grid was immersed in the FTL or FTML sample reservoirs at room temperature, blotted with blotting paper to reduce film thickness and draw the sample into the grid, and vitrified in liquid ethane. The sample was then transferred to liquid nitrogen for storage. Imaging was performed at $200 \mathrm{kV}$ in a stage cooled by liquid nitrogen (Model 915 , Gatan Inc., Pleasonton, CA). Size analysis was performed using ImageJ software using FTL or FTML populations of 50 or greater.

\section{Fluorescence anisotropy}

The fluidity of FTML bilayers was examined by fluorescence anisotropy (Perkin Elmer LS 55) using the hydrophobic bilayer probe diphenylhexatriene (DPH), which was added to the lipid mixture at a 500:1 lipid to DPH ratio prior to thin film hydration. Anisotropy was conducted at $1 \mu \mathrm{M} \mathrm{DPPC}$ from 10 to $70{ }^{\circ} \mathrm{C}$ at a rate of $1^{\circ} \mathrm{C} \mathrm{min}^{-1}$ under continuous mixing. Steady-state DPH anisotropy within the DPPC bilayer was determined at $\lambda_{\mathrm{ex}}=350 \mathrm{~nm}$ and $\lambda_{\mathrm{em}}=452 \mathrm{~nm}$ using the expression $\langle r\rangle=\left(I_{\mathrm{VV}}-I_{\mathrm{VH}}\right) /\left(I \mathrm{vv}+G \times I_{\mathrm{VH}}\right)$ where $I$ represents the emission intensity, the subscripts $\mathrm{V}$ and $\mathrm{H}$ represent the vertical and horizontal orientation of the excitation and emission polarizers, and $G=I_{\mathrm{HV}} / I_{\mathrm{HH}}$ accounts for the sensitivity of the instrument towards vertically and horizontally polarized light. ${ }^{20}$

\section{Doxorubicin release}

DOX release was examined with and without RF-heating using dialysis. FTMLs were loaded at $0.5 \mathrm{ml}$ into $1.5 \mathrm{ml}$ Flotalyzer ${ }^{\mathrm{TM}}$ dialysis tubes with $8 \mathrm{kDa}$ molecular weight cut-off. The dialysis tubes were immersed in $20 \mathrm{ml} 10 \times$ PBS sampling reservoirs. Dialysate sample volumes of $10 \mu \mathrm{l}$ were collected every $15 \mathrm{~min}$ and DOX concentration was measured by fluorescence spectroscopy against a calibration curve (Perkin Elmer LS55, Waltham, MA). Fresh PBS was added to the reservoir to maintain $20 \mathrm{ml}$. For RF-heating, a $2.54 \mathrm{~cm}$ copper 
heating coil containing 3 loops was placed around the sampling reservoir. Heating was conducted for $2 \mathrm{~h}$ at $250 \mathrm{~A}$ and $287 \mathrm{kHz}$ using a $1 \mathrm{~kW}$ Hotshot $^{\mathrm{TM}}$ from Ameritherm Inc. (Scottsville, New York).

This produced an electromagnetic field of $5.9 \times 10^{5} \mathrm{kA} \mathrm{m}^{-1} \mathrm{~s}^{-1}$ based on the product $H f$ where $H$ is the field amplitude (current $\times$ number of coils per length) and $f$ is the frequency. Heating was stopped briefly to retrieve a sample and makeup the dialysate reservoir volume. It is important to note that the dialysis setup was made entirely from plastic or glass, and contained no metal that could have been heated by the field.

The specific absorbance rate (SAR) of the SPIO nanoparticles was determined in DI water at a concentration of $3 \mathrm{mg} \mathrm{ml}^{-1}$ using a fiber optic temperature probe (Luxtron). SAR was determined from the initial slope of temperature as a function of time using the expression $\Delta T / \Delta t=S A R m_{N P} /\left(\overline{c_{p}} m_{V}\right)$ where ${ }^{m}{ }_{N}$ and $m_{V}$ are the mass of the nanoparticles and sample volume, respectively, and $\overline{c_{p}}=\phi c_{p, N}+(1-\phi) c_{p, F}$ is the average heat capacity based on the nanoparticle volume fraction in the sample, $\phi$. The field was identical to that of the DOX release experiment.

\section{In vitro HeLa cell uptake studies}

HeLa and ZR-75-1 (human breast carcinoma) cell lines (American Type Culture Collection, Manassas, VA) were maintained at $37{ }^{\circ} \mathrm{C}$, under humidified air with $5 \% \mathrm{CO}_{2}$, in Dulbecco's Modified Eagle Medium (DMEM) supplemented with 5\% (v/v) fetal bovine serum (FBS), $100 \mathrm{U} \mathrm{mL}^{-1}$ penicillin $\mathrm{G}, 100 \mu \mathrm{g} \mathrm{mL}-1$ streptomycin, $2 \mathrm{mM}$ GlutaMAX, 0.15\% (w/v) sodium bicarbonate, $1 \mathrm{mM}$ sodium pyruvate, $10 \mathrm{mM}$ HEPES buffer, and $100 \mu \mathrm{M}$ nonessential amino acids (cell culture media and additives were from Invitrogen/Life Technologies, Carlsbad, CA). Cells in maintenance medium were trypsinized and seeded into 6-well culture plates at optimum confluence. Incubations were conducted at $1 \mu \mathrm{M}$ FTMLs or $1 \mu \mathrm{M}$ MLs(lipid basis) in serum-containing maintenance medium for 30, 60, 120, or $180 \mathrm{~min}$. At these time points, cells were washed in $1 \times$ PBS to remove free FTMLs or MLs. Cellular uptake was monitored by fluorescence microscopy on an inverted Nikon microscope under $200 \times$ or $400 \times$ magnification. Uptake experiments were conducted in triplicate.

\section{Folate receptor 1 (FOLR1) expression}

FOLR1 expression profile was examined by quantitative PCR. Briefly, total RNA was isolated from cell lines using Trizol reagent or PureLink columns (Invitrogen Corp., Carlsbad, CA) and aliquots were subjected to reverse transcription to make cDNA using High Capacity cDNA kit (Applied Biosystems). FOLR1 and beta-actin specific primers were designed using the webtool QPrimerDepot (http://primerdepot.nci.nih.gov). ${ }^{21}$ Quantitative real-time PCR was performed using SYBR Green master mix, cDNA and genespecific primers (Applied Biosystems) and FOLR1 expression was normalized to beta-actin expression among cell lines.

\section{Results}

\section{FTL and FTML structure and stability}

Co-localization of NBD-PE lipid (green; Figure 1A) and DOX (red; Figure 1B) within FTMLs was verified by fluorescence microscopy. The results show that there was no measurable DOX present outside of the liposomes and that unencapsulated DOX was successfully removed by dialysis. Removal of $96.5 \%$ of the unencapsulated DOX was confirmed after dialysis. With respect to nanoparticle encapsulation, an encapsulation efficiency of $89 \%$ was estimated based on ICP-MS. It should be noted that encapsulation 
efficiency accounts for nanoparticles encapsulated within the aqueous FTML core and bound to the external FTML bilayer surface.

Cryo-TEM analysis showed that FTL and FTMLs exhibited similar size and structure, which indicates that DOX and SPIO nanoparticle encapsulation or binding did not affect FTML formation (Figures 2A and 2B, respectively). Nanoparticle encapsulation was observed within FTMLs (Figure 2B-4, B-5, black arrows). However, encapsulation was heterogeneous as evident from (i) liposomes containing no nanoparticles, (ii) FTMLs containing single nanoparticles, and (iii) FTMLs containing nanoparticle aggregates. The dark structure shown in Figure 2B-5 (black arrow) may depict an unencapsulated nanoparticle aggregate or lipids in cubic ice. ${ }^{22}$ If it was an aggregate, this was the only one observed throughout the TEM grid, consistent with the high encapsulation efficiency (89\%), and would have likely formed due to van der Waals and magnetic attraction ${ }^{23}$ coupled with charge screening of internanoparticle electrostatic repulsion. If present, unencapsulated nanoparticle aggregates could not have been removed by dialysis.

Three distinct liposome structures were observed by cryo-TEM and fluorescence microscopy; large unilamellar liposomes, smaller liposomes encapsulated within larger liposomes, and cup-shaped liposomes (Figure 2A, B). Consistency between cryo-TEM and fluorescence images confirms that the structures were not artifacts of cryo-TEM sample preparation. The cup 'rims' can be clearly seen in the images, which suggests that the cupshaped liposomes formed by single unilamellar liposomes collapsing within themselves and/ or the fusion of inner (encapsulated) liposomes to outer (encapsulating) liposomes. The average diameter of all the structures observed was $174 \pm 53 \mathrm{~nm}$, consistent with membrane extrusion. However, the presence of larger liposomes up to approximately $300 \mathrm{~nm}$ suggests that the liposomes restructured and/or fused during post-extrusion. Fluorescence microscopy images also show liposome cupping and what appears to be budding (Figure 2C).

In addition to size and structure, bilayer fluidity is an important component to liposome function. Fluorescence anisotropy results on the FTMLs show that DPH anisotropy, $\langle r\rangle$, increased with temperature up to $70{ }^{\circ} \mathrm{C}$ (Figure 3). The melting temperature $\left(T_{m}\right)$ of the FTML bilayers was estimated at $1{ }^{\circ} \mathrm{C}$ based on the weighted average of all components (Table 2). Hence, this result indicates that the fluid bilayer become more ordered, or less fluid, with increasing temperature.

\section{FTML doxorubicin release via electromagnetic heating}

Dialysis was conducted with and without RF-heating for $2 \mathrm{~h}$ to determine the extent of spontaneous and nanoparticle-mediated DOX release from the FTMLs. Results are expressed as normalized release based on fluorescence intensities, $I_{\mathrm{RF}} / I_{0}$, to demonstrate the apparent RF-triggered release relative to spontaneous leakage (Figure 4). With RF-heating, a 3 -fold increase in DOX release was observed from 45 to $90 \mathrm{~min}$. The lag from 0 to $45 \mathrm{~min}$ is attributed the time it took DOX to diffuse through the dialysis membrane and into the dialysate after being released from the FTMLs.

\section{FTML uptake by HeLa cells in vitro}

The uptake of FTMLs or MLs (no FR targeting lipid) at $1 \mu \mathrm{M}$ was examined as a function of time by microscopy in plated HeLa (Figure 5) and ZR-75 cell lines (Figure S1, Supplemental Material). In this case the assemblies did not contain DOX because it is cytotoxic. Corresponding brightfield and fluorescence images are shown to provide a qualitative comparison of cellular uptake as a function of time. HeLa cell uptake was observed for both FTMLs and MLs. Based on the observed fluorescence intensity, the degree of uptake was similar up to $120 \mathrm{~min}$. However, at $180 \mathrm{~min}$ the images depict greater 
of the FR-targeted FTMLs compared to the MLs. Closer examination at $60 \mathrm{~min}$ and $180 \mathrm{~min}$ (Figure S2, Supplemental Material) indicate that at $60 \mathrm{~min}$ the FTMLs were bound to the cell surface, while at $180 \mathrm{~min}$ they were internalized. In comparison, no FTML or ML uptake was observed in ZR-75-1 cells. This is result is consistent with FOLR1 expression results, where HeLa cells showed 42.5-fold greater expression than ZR-75-1.

\section{Discussion}

There are a number of reports on FR targeting using liposomes containing folate-lipid conjugates. ${ }^{15,24-28}$ Closely related to this work is that of Pradhan et al, ${ }^{10}$ who designed magnetoliposomes with co-encapsulated $60 \mathrm{~nm}$ magnetic particles and DOX. They were composed of dipalmitoylphosphatidylcholine (DPPC), Chol, $\mathrm{PEG}_{2000}$-DSPE, and Fol$\mathrm{PEG}_{2000}$-DSPE, and exhibited a melting temperature near $41^{\circ} \mathrm{C}$. HeLa cell uptake was demonstrated, similar to our work, and reductions in cell viability were attributed to a synergistic effect of DOX and hyperthermia.

In this work the PEG linker associated with the folate ligand $\left(\mathrm{PEG}_{2000}\right)$ was longer than that of the stabilizing PEG-lipid $\left(\mathrm{PEG}_{750}\right)$. This was chosen to eliminate steric interference of folate binding, which has been demonstrated for stabilizing and folate targeting lipids with the same PEG linker. ${ }^{29}$ Second, the FTMLs were designed to be in the fluid phase at room temperature. Kawano et $\mathrm{al}^{28}$ have shown that greater bilayer fluidity enhances the antitumor activity of FR targeting liposomes. Third, the FTMLs have been prepared with the intent of orienting small, anionic SPIO nanoparticles near the bilayers via electrostatic attraction. Cryo-TEM micrographs showed nanoparticle and nanoparticles aggregates near FTML bilayers, presumably aided by this attraction.

Cryo-TEM revealed a number of FTML structures. Cup-shaped liposomes, denoted by white arrows in Figure 2A and B, are interesting and may have been formed by curvature strain caused by lipid packing (Table 2 ) and/or the fusion of inner to outer liposomes. At the concentrations employed ( $<5$ mol\%), $\mathrm{PEG}_{750}$-DMPE (4 mol\%) and Fol- $\mathrm{PEG}_{2000}$-DSPE $(0.1 \mathrm{~mol} \%)$ likely extended from the liposome/water interface in mushroom configurations and did not interact laterally. ${ }^{30,31}$ The length can be calculated from $L_{m u s h}=a N^{3 / 5}$ where $a$ is the monomer length $(0.35 \mathrm{~nm})$ and $N$ is the degree of polymerization (17 and 46 for 750 and 2000 MW PEG, respectively). ${ }^{30}$ From this equation, $\mathrm{PEG}_{750}$-DMPE and Fol- $\mathrm{PEG}_{2000^{-}}$ DSPE extended approximately $1.9 \mathrm{~nm}$ and $3.5 \mathrm{~nm}$ from the liposome surfaces, respectively. It has been shown that conjugated PEG does not affect lipid packing parameters $(P P)$ at or below $4 \mathrm{~mol} \%$. The major component, DOPC (47.5 mol\%), had a $P P=1.08$ and the cationic lipid, DPTAP $\left(18.9 \mathrm{~mol} \%\right.$ ), had a $P P=0.91$ (average $P P=1.08$ ). ${ }^{32}$ Conical lipids (i.e. $P P<$ 1) in heterogeneous liposomes can phase separate into regions that exhibit lower bending elasticity and, hence, adopt higher curvature. ${ }^{33}$ In the FTMLs, the lower $P P$ and higher melting temperature of DPTAP relative to DOPC may have caused it to phase separate into high curvature regions that supported the cup rims. This would have been aided by charge screening of repulsive DPTAP headgroup interactions at the high electrolyte concentration $(10 \times$ PBS). DPTAP phase separated would have increased the average $P P$ of the remaining bilayer $(P P \sim 1.1)$ to favor negative curvature, which was observed on the inner cup bilayer. ${ }^{34}$ It is important to note that the observed structures are likely dynamic and may undergo a number of shape fluctuations.

In addition to curvature strain, fusion may have been promoted by the presence PEG-lipids, and the fusion of inner to outer liposomes may have contributed to liposome cupping. As the inner liposomes approached the outer, the point of impending contact may have been devoid of PEG which produces an osmotic pressure that drives liposome aggregation. ${ }^{35}$ Yang et $\mathrm{al}^{36}$ have shown that large unilamellar liposomes can aggregate with $1-2 \mathrm{~mol} \%$ PEG-lipid 
and that curvature stress within the bilayers, inferred in this work from the presence of cupshaped liposomes, can lead to liposome fusion. Furthermore, Varga et $\mathrm{al}^{37}$ have shown that PEG-lipids (in their case $\mathrm{PEG}_{2000}$-DSPE) exhibit an asymmetric distribution across the bilayer ( $75 \%$ outside), which can also enhance curvature strain. While we did not observe aggregation, it is possible that PEG-lipids produced additional curvature strain within the FTML bilayers and caused fusion. Budding, observed by cryo-TEM and fluorescence microscopy, is also driven by these factors.

Despite the evidence of FTML restructuring, qualitative evidence suggests that this did not compromise their stability. This is intuitive considering that the PEG-lipids would provide steric stabilization despite being involved in restructuring. Fluorescence microscopy showed that the FTMLs remained dispersed in 10x PBS. This is consistent with cryo-TEM analysis, which showed no FTML aggregation. Furthermore, there was no evidence of sedimentation after two weeks of storage at room temperature (results not shown). This is an important observation as it demonstrates that the addition of oppositely charged nanoparticles to cationic liposomes does not cause heteroaggregation.

Anisotropy results confirmed that the FTMLs existed in a fluid state (as expected). However, the increase in anisotropy with temperature reflected a decrease in local bilayer fluidity that can be attributed to the effects of PEG on lipid hydration. It is known that water becomes a 'poorer' solvent for PEG with increasing temperature, which increases PEG hydrophobicity. ${ }^{38}$ In turn, increasing PEG hydrophobicity would also reduce lipid hydration. The anisotropy results are consistent with previous work that has shown that the inclusion of PEG-lipid in fluid liposomes (below $8 \mathrm{~mol} \%$ ) leads to greater adiabatic compressibility and reduced lipid hydration with increasing temperature. ${ }^{39}$ Changes in bilayer compressibility would have also enhanced curvature strain.

While cryo-TEM results showed that nanoparticle encapsulation had little affect on FTML structure, DOX release results demonstrated that RF-heating of the nanoparticles did played an active role in triggering release. However, we cannot directly attribute this to nanoparticle heating due to the fact that the average $T_{\mathrm{m}}$ was $1{ }^{\circ} \mathrm{C}$. Hence, the lipid bilayers were in a fluid state and heating did not trigger a phase transition. Based on a measured $S A R$ value of 98.7 $\mathrm{W} \mathrm{g}{ }^{-1} \gamma-\mathrm{Fe}_{2} \mathrm{O}_{3}$, a temperature change of $9{ }^{\circ} \mathrm{C}$ was estimated over $120 \mathrm{~min}$ (the duration of the release experiment). However, SAR is based on the initial heating slope and this temperature change represents a maximum under adiabatic conditions. Furthermore, this temperature change would have caused only a minor decrease in bilayer fluidity (Figure 3). Recent theoretical ${ }^{40}$ and experimental ${ }^{41,42}$ work has shown that the temperature difference between a nanoparticle surface its surroundings due to electromagnetic field heating (light or $\mathrm{RF}$ ) is negligible due to rapid heat dissipation from the nanoparticle surface. While FTML heating may have contributed to DOX release, mechanically-induced release due to the rotation or oscillation of nanoparticles bound to the lipid bilayer may be more likely.

It is important to note that the FTMLs were designed as a base structure for combined targeting and controlled drug release via an external RF trigger. The lipid composition, nanoparticle loading, drug concentration, or RF field conditions can be modified to optimize performance. Preliminary results demonstrate that FTMLs are capable of binding to and being internalized by FR-expressing HeLa cells. Uptake through FR binding is attributed to FR-mediated endocytosis, ${ }^{16}$ which was confirmed by comparing FTML verses ML uptake, and HeLa verses ZR-75-1 uptake. Future work is needed to quantify the extent of binding and uptake, and FTML stability in serum-containing medium.

The therapeutic efficacy of nanoscale assemblies is tied to the ability to (i) prevent premature drug release before reaching a target site, (ii) initiate or "trigger" drug release on 
demand once a target site has been reached, and (iii) control the rate of drug release to the target site. This work has demonstrated that FTMLs can be designed with these properties by co-encapsulating DOX and SPIO nanoparticles. The FTMLs displayed solution structures consistent with lipid packing parameters and the effects of PEGylated lipids on liposome structure. Fluorescence anisotropy results depicted a decrease in bilayer fluidity with increasing temperature, consistent with greater PEG hydrophobicity and lipid dehydration. While FTMLs did exhibit colloidal stability over the conditions examined, additional work is required to assess the long-term stability, and the effects of biologically relevant serum protein concentrations and increased nanoparticle loading on this stability. Furthermore, thought nanoparticle encapsulation was achieved, optimization is required to improve the extent and efficiency of encapsulation. This, ultimately, can be used to control the drug release properties via RF-heating.

\section{Supplementary Material}

Refer to Web version on PubMed Central for supplementary material.

\section{Acknowledgments}

This research was supported by the National Science Foundation (NSF, Grant no. CBET-0931875) and by RIINBRE (Grant no. P20RR016457) from the National Center for Research Resources (NCRR), a component of the National Institutes of Health (NIH). Its contents are solely the responsibility of the authors and do not necessarily represent the official views of NSF, NCRR, or NIH. The authors declare no competing interests.

\section{References}

1. Al-Jamal WT, Kostarelos K. Liposome-nanoparticle hybrids for multimodal diagnostic and therapeutic applications. Nanomed. 2007; 2:85-98.

2. Bothun GD. Hydrophobic silver nanoparticles trapped in lipid bilayers: Size distribution, bilayer phase behavior, and optical properties. J Nanobiotechnology. 2008; 6:13. [PubMed: 19014492]

3. Gopalakrishnan G, Danelon C, Izewska P, Prummer M, Bolinger PY, Geissbuhler I, et al. Multifunctional lipid/quantum dot hybrid nanocontainers for controlled delivery of live cells. Angew Chem Int Ed. 2006; 45:5478-83.

4. Volodkin DV, Skirtach AG, Mohwald H. Near-IR Remote Release from Assemblies of Liposomes and Nanoparticles. Angew Chem Int Ed. 2009; 48:1807-09.

5. De Cuyper M, Joniau M. Magnetoliposomes: Formation and structural characterization. Eur Biophys J. 1988; 15:311-19. [PubMed: 3366097]

6. Soenen SJH, Hodenius M, De Cuyper M. Magnetoliposomes: versatile innovative nanocolloids for use in biotechnology and biomedicine. Nanomed. 2009; 4:177-91.

7. Babincova M, Sourivong P, Chorvat D, Babinec P. Laser triggered drug release from magnetoliposomes. J Magn Magn Mater. 1999; 194:163-66.

8. Viroonchatapan E, Sato H, Ueno M, Adachi I, Tazawa K, Horikoshi I. Release of 5-fluorouracil from thermosensitive magnetoliposomes induced by an electromagnetic field. J Control Release. 1997; 46:263-71.

9. Chen YJ, Bose A, Bothun GD. Controlled Release from Bilayer-Decorated Magnetoliposomes via Electromagnetic Heating. ACS Nano. 2010; 4:3215-21. [PubMed: 20507153]

10. Pradhan P, Giri J, Rieken F, Koch C, Mykhaylyk O, Doblinger M, et al. Targeted temperature sensitive magnetic liposomes for thermo-chemotherapy. J Control Release. 2010; 142:108-21. [PubMed: 19819275]

11. Dandamudi S, Campbell RB. Development and characterization of magnetic cationic liposomes for targeting tumor microvasculature. BBA-Biomembranes. 2007; 1768:427-38. [PubMed: 17258172]

12. Soenen SJH, Brisson AR, De Cuyper M. Addressing the problem of cationic lipid-mediated toxicity: The magnetoliposome model. Biomaterials. 2009; 30:3691-701. [PubMed: 19371948] 
13. Brunner TJ, Wick P, Manser P, Spohn P, Grass RN, Limbach LK, et al. In vitro cytotoxicity of oxide nanoparticles: Comparison to asbestos, silica, and the effect of particle solubility. Environ Sci Technol. 2006; 40:4374-81. [PubMed: 16903273]

14. Limbach LK, Li Y, Grass RN, Brunner TJ, Hintermann MA, Muller M, et al. Oxide nanoparticle uptake in human lung fibroblasts: Effects of particle size, agglomeration, and diffusion at low concentrations. Environ Sci Technol. 2005; 39:9370-76. [PubMed: 16382966]

15. Lee RJ, Low PS. Delivery of liposomes into cultured KB cells via folate receptor-mediated endocytosis. J Biol Chem. 1994; 269:3198-204. [PubMed: 8106354]

16. Sabharanjak S, Mayor S. Folate receptor endocytosis and trafficking. Adv Drug Deliv Rev. 2004; 56:1099-109. [PubMed: 15094209]

17. Duan HW, Kuang M, Wang XX, Wang YA, Mao H, Nie SM. Reexamining the effects of particle size and surface chemistry on the magnetic properties of iron oxide nanocrystals: New insights into spin disorder and proton relaxivity. J Phys Chem C. 2008; 112:8127-31.

18. Smith AM, Duan HW, Rhyner MN, Ruan G, Nie SM. A systematic examination of surface coatings on the optical and chemical properties of semiconductor quantum dots. Physical Chemistry Chemical Physics. 2006; 8:3895-903. [PubMed: 19817050]

19. Pradhan P, Giri J, Banerjee R, Bellage J, Bahadur D. Preparation and characterization of manganese ferrite-based magnetic liposomes for hyperthermia treatment of cancer. J Magn Magn Mater. 2007; 311:208-15.

20. Lakowicz, JR. Fluorescence Anisotropy, in Principles of Fluorescence Spectroscopy. 2nd. Kluwer Academic; New York: 1999.

21. Cui W, Taub DD, Gardner K. qPrimerDepot: a primer database for quantitative real time PCR. Nucleic Acids Res. 2007; 35:D805-9. [PubMed: 17068075]

22. Friedrich H, Frederik PM, de With G, Sommerdijk NAJM. Imaging of Self-Assembled Structures: Interpretation of TEM and Cryo-TEM Images. Angewandte Chemie-International Edition. 2010; 49:7850-58.

23. Mefford OT, Vadala ML, Goff JD, Carroll MRJ, Mejia-Ariza R, Caba BL, et al. Stability of polydimethylsiloxane-magnetite nanoparticle dispersions against flocculation: Interparticle interactions of polydisperse materials. Langmuir. 2008; 24:5060-69. [PubMed: 18366222]

24. Gabizon A, Shmeeda H, Horowitz AT, Zalipsky S. Tumor cell targeting of liposome-entrapped drugs with phospholipid-anchored folic acid-PEG conjugates. Adv Drug Deliv Rev. 2004; 56:1177-92. [PubMed: 15094214]

25. Xiang G, Wu J, Lu Y, Liu Z, Lee RJ. Synthesis and evaluation of a novel ligand for folatemediated targeting liposomes. Int J Pharm. 2008; 356:29-36. [PubMed: 18258394]

26. Saul JM, Annapragada A, Natarajan JV, Bellamkonda RV. Controlled targeting of liposomal doxorubicin via the folate receptor in vitro. J Control Release. 2003; 92:49-67. [PubMed: 14499185]

27. Gabizon A, Tzemach D, Gorin J, Mak L, Amitay Y, Shmeeda H, et al. Improved therapeutic activity of folate-targeted liposomal doxorubicin in folate receptor-expressing tumor models. Cancer Chemoth Pharm. 2010; 66:43-52.

28. Kawano K, Onose E, Hattori Y, Maitani Y. Higher Liposomal Membrane Fluidity Enhances the in Vitro Antitumor Activity of Folate-Targeted Liposomal Mitoxantrone. Mol Pharmaceut. 2009; 6:98-104.

29. Gabizon A, Horowitz AT, Goren D, Tzemach D, Mandelbaum-Shavit F, Qazen MM, et al. Targeting folate receptor with folate linked to extremities of poly(ethylene glycol)-grafted liposomes: In vitro studies. Bioconjugate Chem. 1999; 10:289-98.

30. Hristova K, Needham D. The Influence of Polymer-Grafted Lipids on the Physical-Properties of Lipid Bilayers - a Theoretical-Study. J Coll Interfac Sci. 1994; 168:302-14.

31. Garbuzenko O, Barenholz Y, Priev A. Effect of grafted PEG on liposome size and on compressibility and packing of lipid bilayer. Chem Phys Lipids. 2005; 135:117-29. [PubMed: 15921973]

32. Junquera E, Arranz R, Aicart E. Mixed vesicle formation on a ternary surfactant system: Didodecyldimethylammonium bromide/dodecylethyldimethylammonium bromide/water. Langmuir. 2004; 20:6619-25. [PubMed: 15274564] 
33. Lipowsky R. The Morphology of Lipid-Membranes. Curr Opin Struc Biol. 1995; 5:531-40.

34. Israelachvili, J. Intermolecular \& surface forces. 2nd. Academic Press; 1991.

35. Kuhl T, Guo YQ, Alderfer JL, Berman AD, Leckband D, Israelachvili J, et al. Direct measurement of polyethylene glycol induced depletion attraction between lipid bilayers. Langmuir. 1996; 12:3003-14.

36. Yang QL, Guo YQ, Li LH, Hui SW. Effects of lipid headgroup and packing stress on poly(ethylene glycol)-induced phospholipid vesicle aggregation and fusion. Biophys J. 1997; 73:277-82. [PubMed: 9199792]

37. Varga Z, Berenyi S, Szokol B, Orfi L, Keri G, Petak I, et al. A Closer Look at the Structure of Sterically Stabilized Liposomes: A Small-Angle X-ray Scattering Study. J Phys Chem B. 2010; 114:6850-54. [PubMed: 20429570]

38. Karlstrom G, Engkvist O. Theory of poly(ethylene glycol) in solution. Acs Sym Ser. 1997; 680:16-30.

39. Tirosh O, Barenholz Y, Katzhendler J, Priev A. Hydration of polyethylene glycol-grafted liposomes. Biophys J. 1998; 74:1371-79. [PubMed: 9512033]

40. Keblinski P, Cahill DG, Bodapati A, Sullivan CR, Taton TA. Limits of localized heating by electromagnetically excited nanoparticles. J Appl Phys. 2006:100.

41. Bothun GD, Priess MR. Bilayer heating in magnetite nanoparticle-liposome dispersions via fluorescence anisotropy. J Coll Inter Sci. 2011 in press.

42. Gupta A, Kane RS, Borca-Tasciuc DA. Local temperature measurement in the vicinity of electromagnetically heated magnetite and gold nanoparticles. J Appl Phys. 2010:108. 

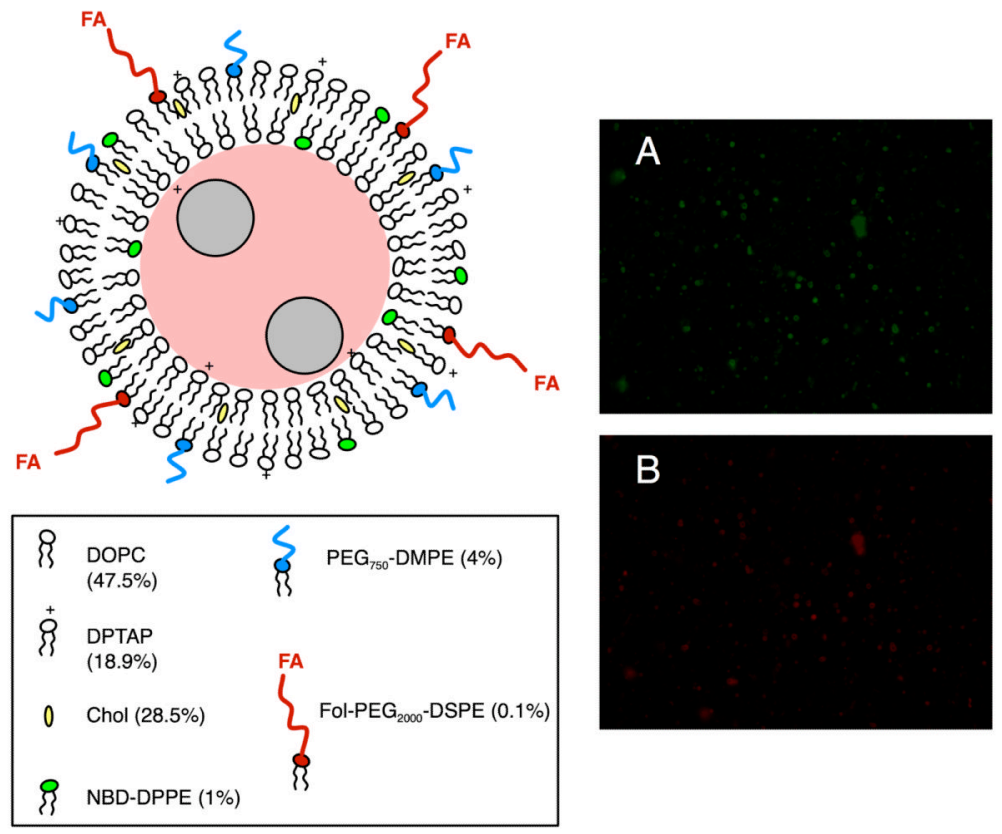

Figure 1.

Schematic of FTMLs containing co-encapsulated doxorubicin (DOX, red) and SPIO nanoparticles (gray). The liposome components include (in order of decreasing molar concentration) zwitterionic 1,2-dioleoyl-sn-glycero-3-phosphocholine (DOPC), cationic 1,2dipalmitoyl-3-trimethylammonium-propane (DPTAP), cholesterol (Chol), PEGylated 1,2dimyristoyl-sn-glycero-3-phosphoethanolamine-N-[methoxy(polyethylene glycol)-750] (PEG-DMPE), fluorescent 1,2-dipalmitoyl-sn-glycero-3-phosphoethanolamine-N-(7nitro-2-1,3-benzoxadiazol-4-yl) (NBD-DPPE), and folate-modified 1,2-distearoyl-snglycero-3-phosphoethanolamine-N-[folate(polyethylene glycol)-2000] (Fol-PEG $2000^{-}$ DSPE). Fluorescence microscopy images of FTMLs in PBS (400× magnification) are shown containing NBD-DPPE (green, A) and encapsulated DOX (red, B). 

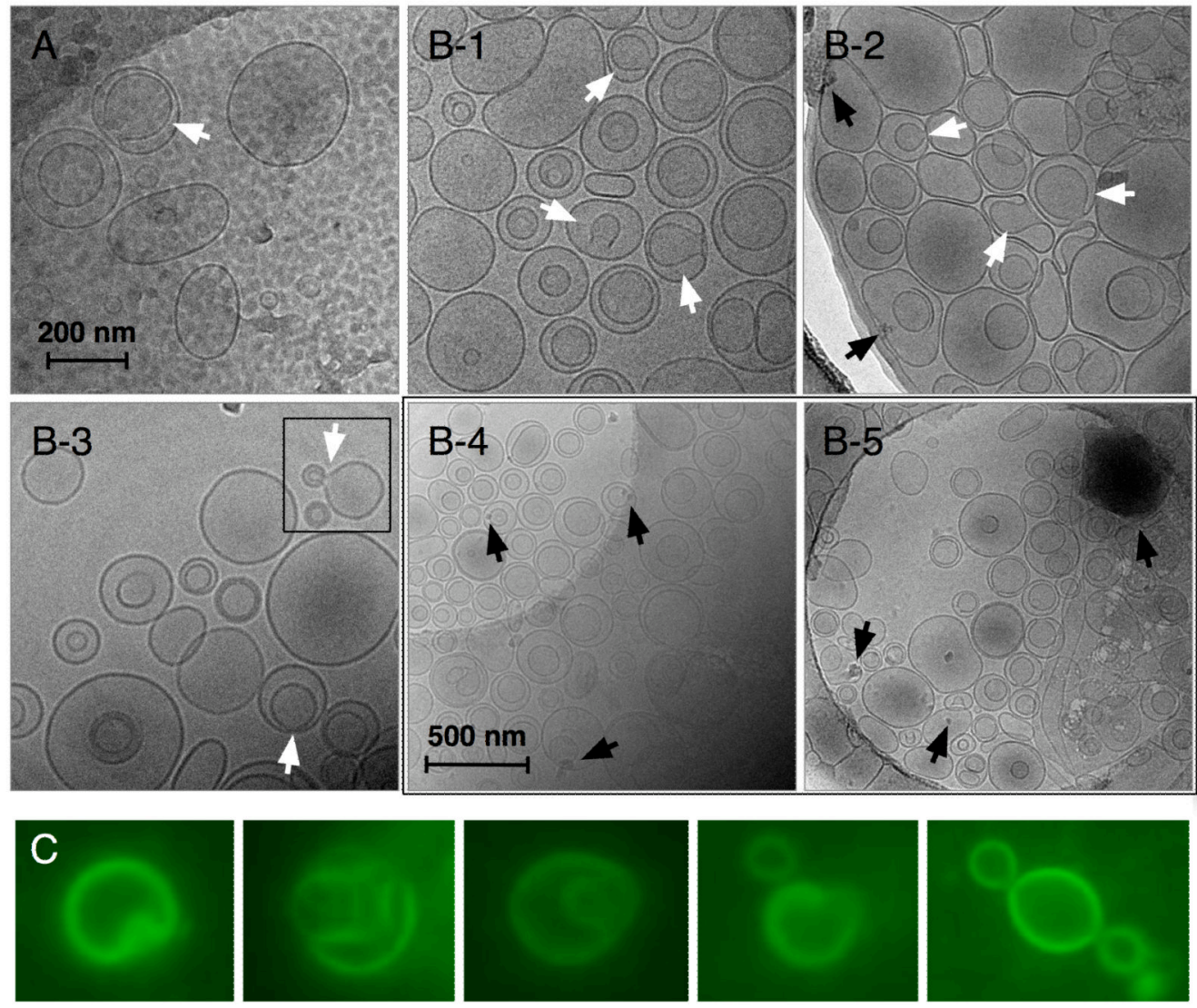

cupping

budding

Figure 2.

Cryo-TEM micrographs of (A) FTLs without encapsulated DOX and (B-1 to B-5) FTMLs containing encapsulated DOX and SPIO nanoparticles. Both samples were prepared by thin film hydration in 10x PBS (pH 7.4) followed by membrane extrusion at $200 \mathrm{~nm}$. The 200 $\mathrm{nm}$ scale bar is common to A and B-1 to B-3, and the $500 \mathrm{~nm}$ scale bare is common to B-4 and B-5. White arrows denote cup-shaped liposomes containing a visible pore or mouth and black arrows denote SPIO nanoparticles and nanoparticle aggregates. The square region denotes liposome budding. Representative magnified fluorescence microscopy images at $1000 \times$ magnification (bottom) of FTML cupping and budding. 


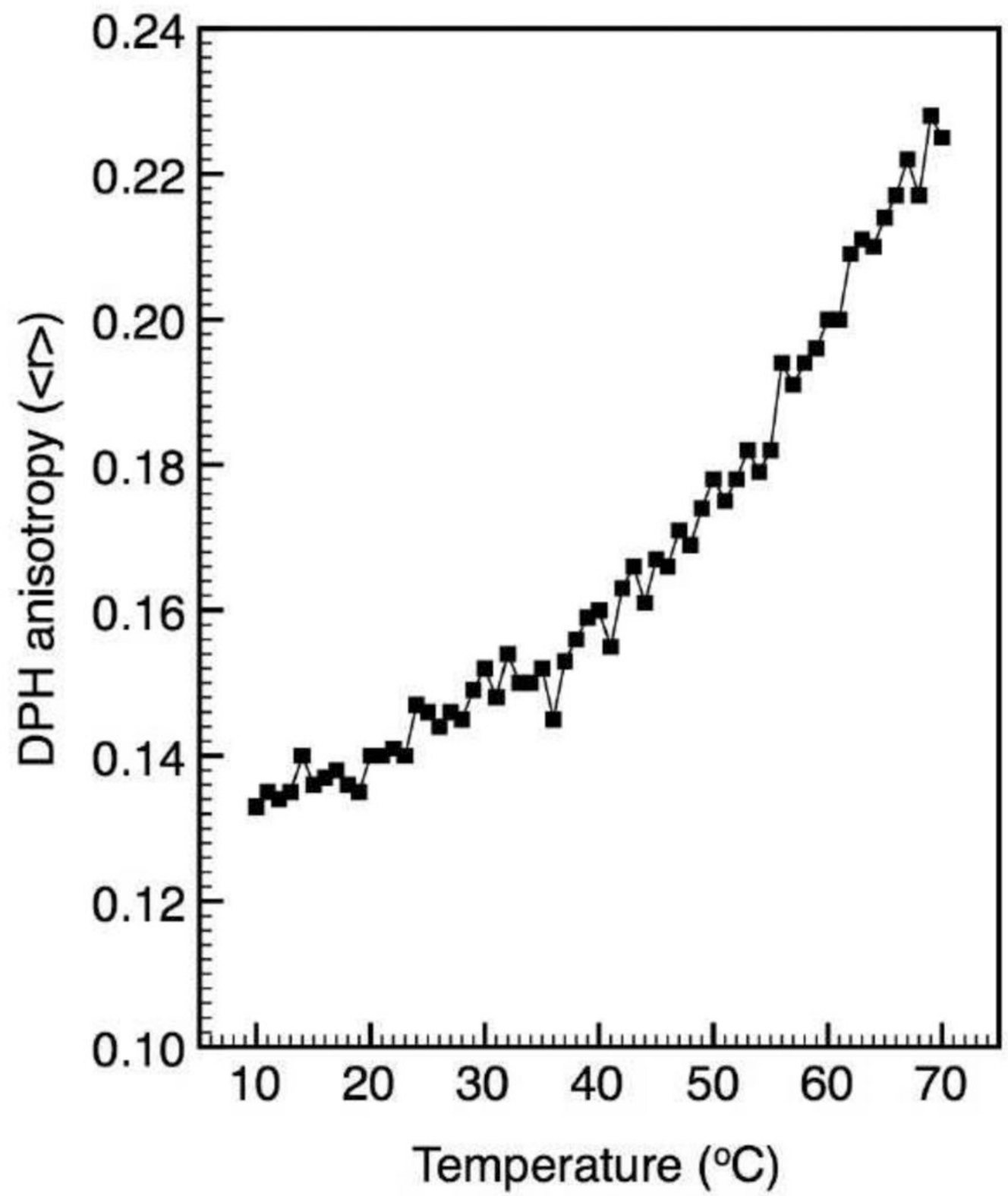

Figure 3.

Fluorescence anisotropy of DPH $(\langle r\rangle)$ in FTML bilayers as a function of increasing temperature. The can rate was $1{ }^{\circ} \mathrm{C} \mathrm{min}-1$. 


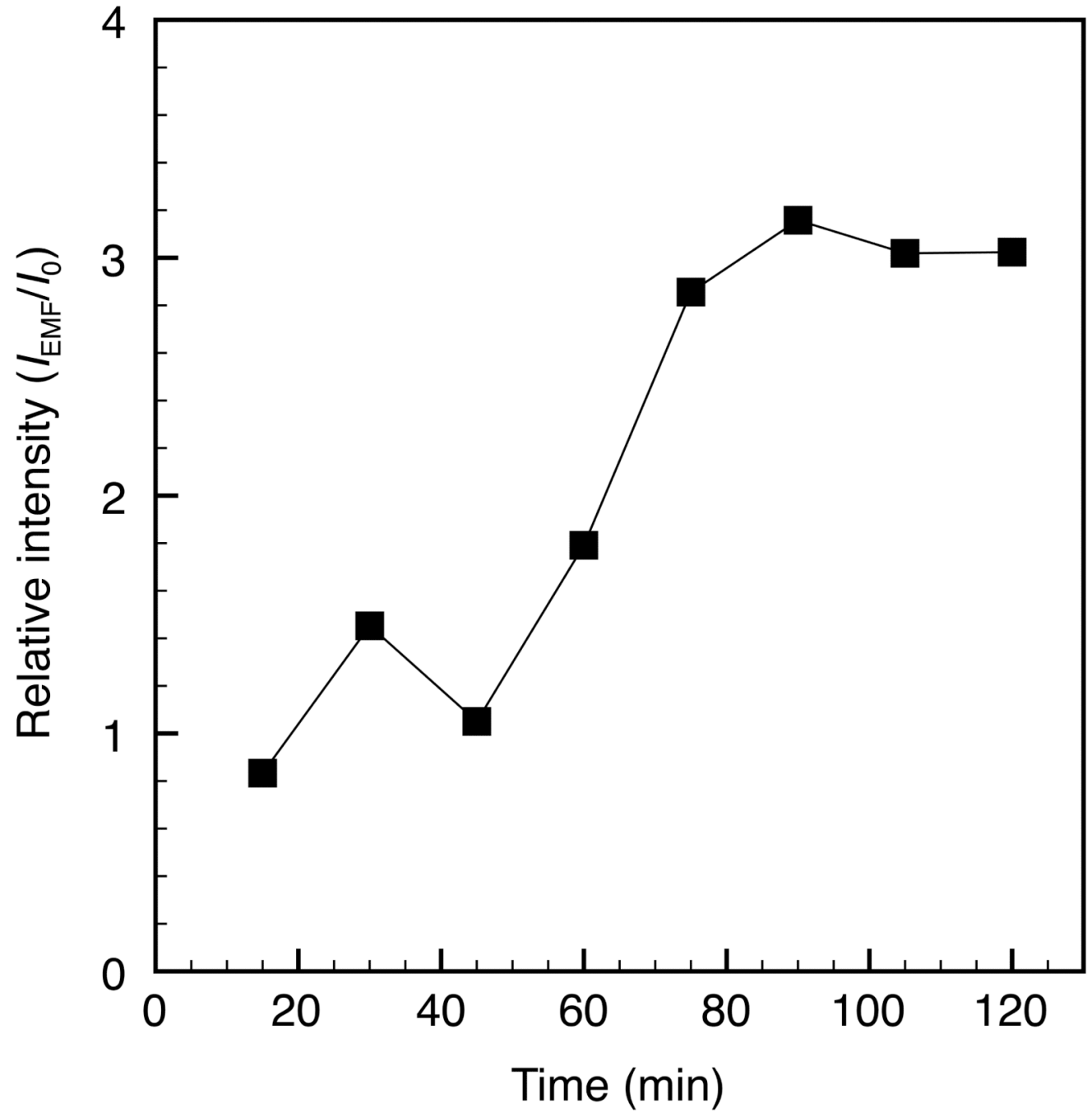

Figure 4.

DOX release from FTMLs based on normalized fluorescence intensities with $\left(I_{\mathrm{RF}}\right)$ and without $\left(I_{0}\right)$ RF-heating for 120 min at a field strength of $5.9 \times 10^{5} \mathrm{kA} \mathrm{m}^{-1} \mathrm{~s}^{-1}$. 


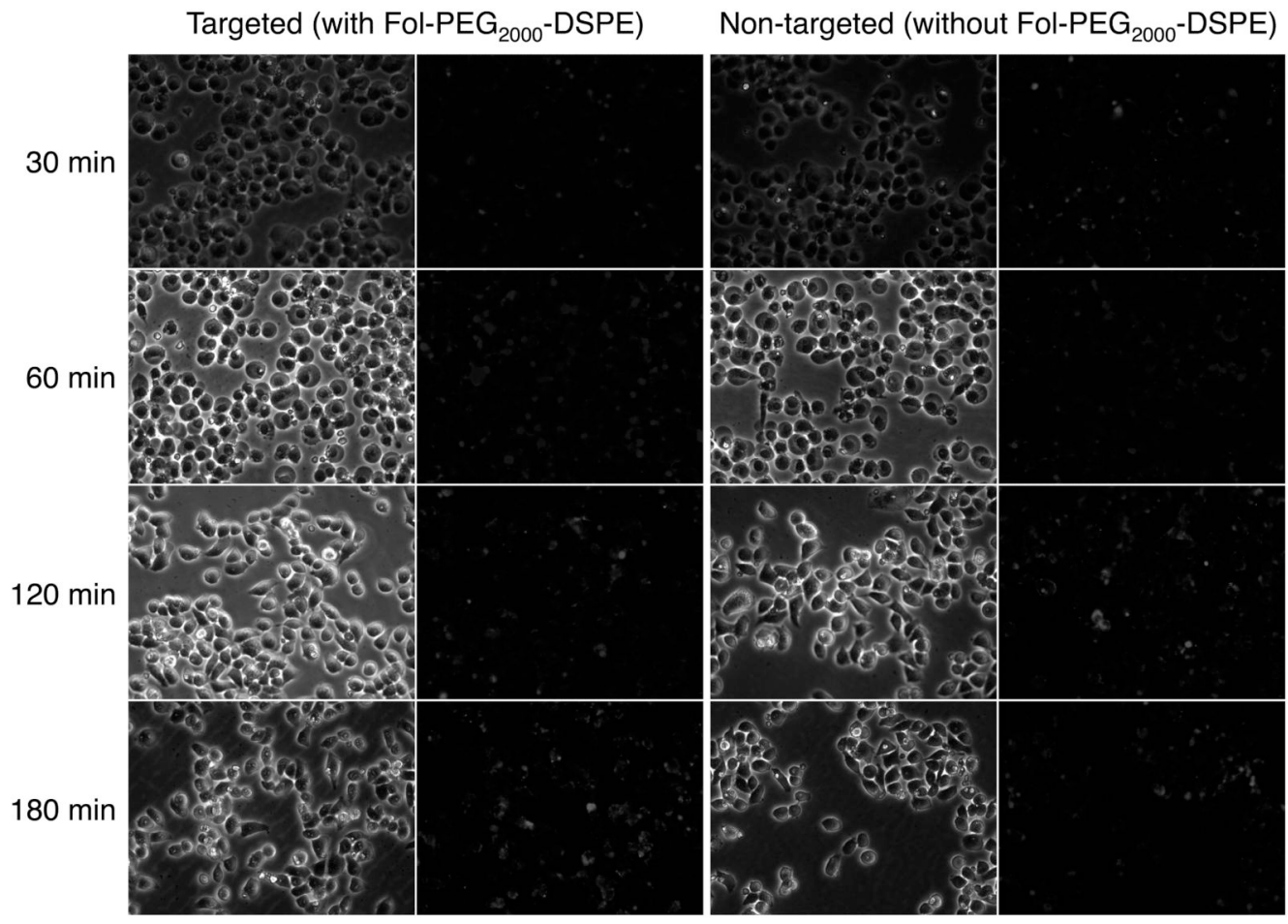

Figure 5.

Brightfield and fluorescence micrographs of in vitro HeLa cell uptake of FTMLs or MLs without DOX (200× magnification) incubated for 30, 60, 120, or $180 \mathrm{~min}$. The green fluorescence is from NBD-DPPE. 
Table 1

Bilayer composition of foltate receptor-targeting magnetoliposomes (FTMLs).

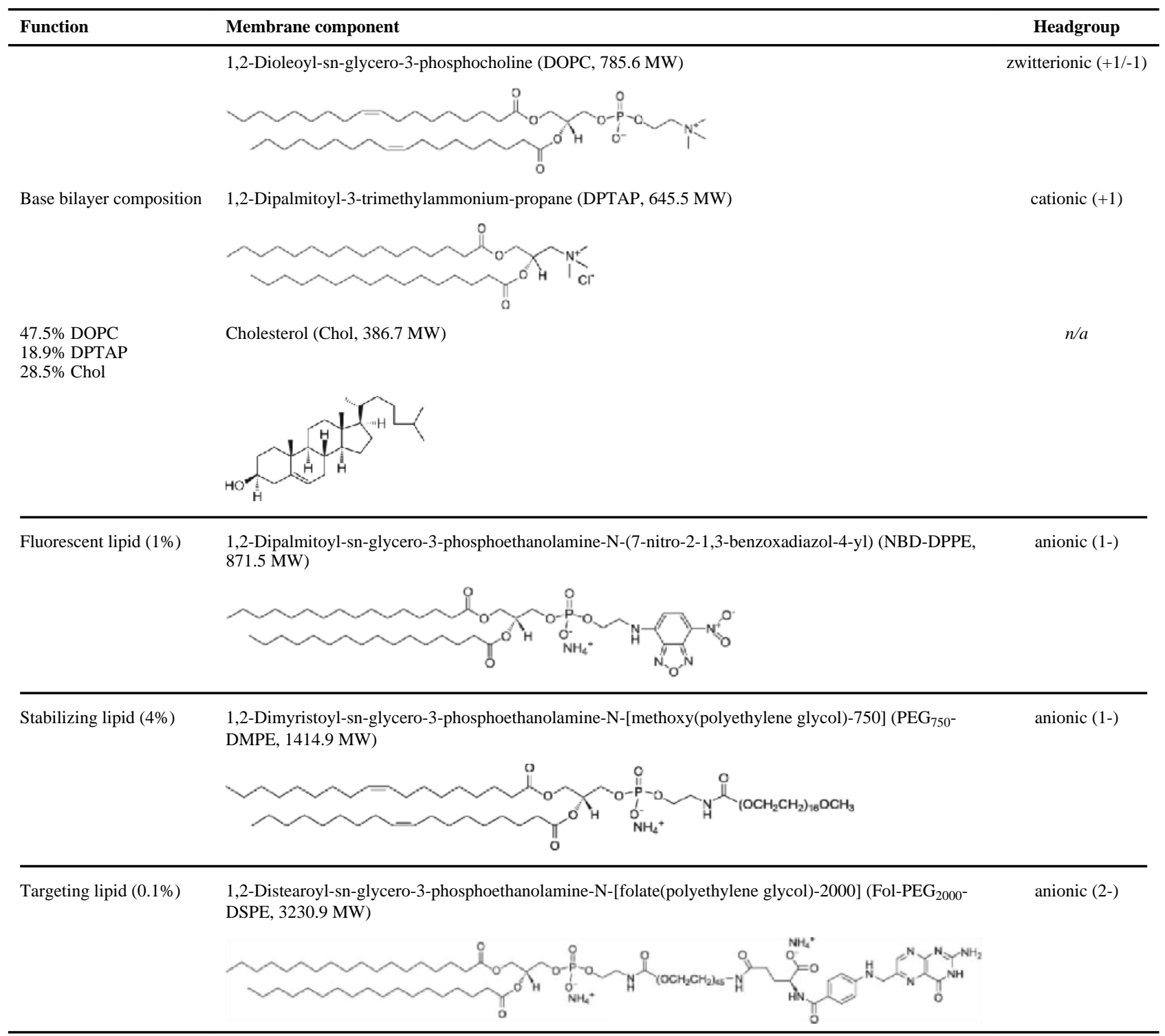


Table 2

Lipid melting temperatures and packing parameters $(P P)$.

\begin{tabular}{lccc}
\hline Membrane component & $\%$ & $\mathbf{T}_{\mathbf{m}}\left({ }^{\circ} \mathbf{C}\right)$ & $\boldsymbol{P P}$ \\
DOPC & $\mathbf{4 7 . 5}$ & $\mathbf{- 2 0}$ & $\mathbf{1 . 0 8}^{\mathbf{2 4}}$ \\
\hline DPTAP & 18.9 & 42 & $0.91^{25}$ \\
Chol & 28.5 & $n / a$ & $1.21^{26}$ \\
NBD-DPPE & 1.0 & $63^{a}$ & $1.04^{b}$ \\
PEG $_{750}-D M P E$ & 4.0 & $50^{a}$ & $1.04^{b}$ \\
Fol-PEG $_{2000}-\mathrm{DSPE}$ & 0.1 & $74^{a}$ & $1.04^{27}$ \\
molar average & & 1 & 1.08 \\
\hline
\end{tabular}

$a_{\text {based on }} \mathrm{T}_{\mathrm{m}}$ of DPPE, DMPE, and DSPE.

${ }^{b}$ Calculated $P P$ based on DSPE, $P P=v_{C}\left(a l_{C}\right)^{-1}$, where $v_{C}$ is the volume of the carbon tails, $a$ is the area per lipid, and $l_{C}$ is the length of the carbon tails. 